\title{
Solution of the Falkner-Skan wedge flow by a revised optimal homotopy asymptotic method
}

\author{
A. G. Madaki ${ }^{*}$, M. Abdulhameed ${ }^{2}$, M. Ali ${ }^{1}$ and R. Roslan ${ }^{1}$
}

\author{
*Correspondence: \\ abdulmdk119@gmail.com \\ ${ }^{1}$ Centre for Research \\ in Computational \\ Mathematics Faculty \\ of Science, Technology \\ and Human Development, \\ Universiti Tun Hussein Onn \\ Malaysia, 86400 Batu Pahat, \\ Johor, Malaysia \\ Full list of author information \\ is available at the end of the \\ article
}

\begin{abstract}
In this paper, a revised optimal homotopy asymptotic method (OHAM) is applied to derive an explicit analytical solution of the Falkner-Skan wedge flow problem. The comparisons between the present study with the numerical solutions using (fourth order Runge-Kutta) scheme and with analytical solution using HPM-Padé of order [4/4] and order [13/13] show that the revised form of OHAM is an extremely effective analytical technique.
\end{abstract}

\section{Background}

In the last few decades, the problems that consist of nonlinear terms have grabbed the interest of many researchers because of its challenging to handle. Many efforts have been made by many researchers to investigate the concrete solutions to such problem of nonlinear differential equations. Due to the fact that, many nonlinear problems do not have a small parameter, so this is what has confined many analytical techniques, among which we have a perturbation technique, and other traditional methods which require the presence of a small parameter in the equation (Nayfeh and Mook 1979). We consider the two-dimensional incompressible laminar boundary layer equations, which are expressed in the form of nonlinear third-order ordinary differential equations as follows.

$$
f^{\prime \prime \prime}+f^{\prime \prime}+\beta\left(1-f^{\prime 2}\right)=0
$$

subject to the boundary conditions

$$
f(0)=0, \quad f^{\prime}(0)=0, \quad f^{\prime}(+\infty)=1 .
$$

This equation was first stated by Falkner and Skan (1931). Though, later on their solutions and dependency on the parameter $\beta$ were investigated by Hartree (1937). The solutions of Falkner-Skan equation have been analytically investigated by many scholars. Abbasbandy and Hayat (2009) studied the solution of the magnetohydrodynamic (MHD) Falkner-Skan flow by homotopy analysis method (HAM). They found that the value of skin friction increases with the increase of magnetic field parameter, while the boundary layer thickness decreases. Yao (2009) investigated the temperature distribution in

(c) 2016 Madaki et al. This article is distributed under the terms of the Creative Commons Attribution 4.0 International License (http://creativecommons.org/licenses/by/4.0/), which permits unrestricted use, distribution, and reproduction in any medium, provided you give appropriate credit to the original author(s) and the source, provide a link to the Creative Commons license, and indicate if changes were made. 
the Falkner-Skan wedge flow using HAM. Analytical solutions of momentum and heat transfer of the Falkner-Skan flow with algebraic decay have been studied by Fang et al. (2012). They observed that the value of the flow controlling parameter, $b$ decreases with the decrease of the wall movement parameter, $\lambda$. Although, the value of the wall mass transfer, $\gamma$ first decreases and then increases, while the wall temperature gradient increases with the decrease of pole number, $n$ and the increase of Prandtl number, Pr. Hayat et al. (2011) studied the porous medium and mixed convection of FalknerSkan wedge flow of a power-law fluid using HAM, their results show that dimensionless velocity distribution decreases with the increase in $P r$. It was observed that the velocity profile increases when the Reynolds number, Re is increased. Yao and Chen (2009) found the series solution to the Falkner-Skan equation with stretching boundary using HAM and compared their results numerically by fourth-order Runge-Kutta method combined with Newton-Raphson technique. A good agreement was found between both approximate analytical solution and numerical result. A numerical method for the solution of the Falkner-Skan equation was investigated using a shooting method by Asaithambi (1997). Asaithambi (1997) was able to obtain the accelerating, constant, decelerating, and reverse flows numerically. The solution of the Falkner-Skan equation for wedge using Adomian decomposition method (ADM) by Alizadeh et al. (2009) indicated that the percentage of error decreases by increasing the number of the ADM terms. Rajagopal et al. (1983) investigated the Falkner-Skan boundary layer flow of a homogeneous incompressible second grade fluid past a wedge placed symmetrically with respect to the fluid flow direction. Moreover, many researchers devoted themselves on investigating the problem of Falkner-Skan flow (Kuo 2005; Pantokratoras 2006; Ishak et al. 2009; Zhu et al. 2009; Rosales-Vera and Valencia 2010; Hsiao 2011; Yacob et al. 2011; Parand et al. 2011; Abdulhameed et al. 2015).

The aim of this paper is to obtain an explicit analytical solution of the Falkner-Skan equation by using a revised OHAM which introduces another function in the auxiliary function in the original OHAM (Marinca and Herisanu 2008, 2014) which is quite good enough to handle the strong challenges in nonlinear differential equations. Recently, Herisanu et al. (2015) studied an analytical approach to non-linear dynamical model of a permanent magnet synchronous generator using OHAM. They studied four different cases at various moments of inertia and electrical resistances specific to sudden short circuit produced at the generator terminals and sudden change of load. They found very promising results when validated with numerical solutions. However, this study will be compared with the numerical solution as well as the solutions by Bararnia et al. (2012) for the acceleration flow $(\beta>0)$ and Rajagopal et al. (1983).

\section{Basic idea of revised OHAM (Marinca and Herisanu 2008, 2014)}

Here OHAM to the differential equation as follows:

$$
L(f(z))+g(z)+N(f(z))=0, \quad B\left(f, \frac{d f}{d z}\right)=0
$$

where $L$ is a linear operator, $z$ is an independent variable, $f(z)$ is an unknown function, $g(z)$ is a known function, $N(f(z))$ is a nonlinear operator and $B$ is a boundary operator. 
By means of OHAM one first constructs a family of equations:

$$
\begin{gathered}
(1-p)[L(\phi(z, p))+g(z)]=H(p, z)[L(\phi(z, p))+g(z)+N(\phi(z, p))], \\
B\left(\phi(z, p), \frac{\partial \phi(z, p)}{d z}\right)=0
\end{gathered}
$$

where $p \in[0,1]$ is an embedding parameter, $L$ is a linear operator which depends on the boundary operator $B$ and on the initial approximation $f_{0}, H(p, z)$ is a nonzero auxiliary function for $p \neq 0$, here $H(0, z)=0$ and $\phi(z, p)$ is an unknown function, respectively. Obviously, when $p=0$ or $p=1$ it holds

$$
\phi(z, 0)=f_{0}(z), \quad \text { or } \quad \phi(z, 1)=f(z) .
$$

As $p$ increases from 0 to 1 , the solution $\phi(z, p)$ varies from $f_{0}(z)$ to the solution $f(z)$, where $f_{0}(z)$ is obtained from Eq. (4) for $p=0$ :

$$
L\left(f_{0}(z)\right)+g(z)=0, \quad B\left(f_{0}, \frac{d f_{0}}{d z}\right)=0 .
$$

Instead of written the auxiliary function as $H(p)=p C_{1}+p^{2} C_{2}+\cdots$, we choose the auxiliary function $H(p, z)$ in the form

$$
H(p, z)=p h_{1}\left(z, C_{1 i}\right)+p^{2} h_{2}\left(z, C_{2 i}\right)+\cdots+p^{m} h_{m}\left(z, C_{m i}\right),
$$

where $h_{1}, h_{2}, \ldots, h_{m}$ are functions depending on the variable $z$ and convergence-control parameters $C_{1 i}, C_{2 i}, \ldots, C_{m i}$ for $i=1,2, \ldots$, which can be determined later. Let us consider the solution of Eq. (4) is in the form

$$
\phi\left(z, p, h_{i}\right)=f_{0}(z)+\sum_{k \geq 1} f_{k}\left(z, h_{i}\right) p^{k}, \quad i=1,2, \ldots,
$$

Substituting Eq. (8) into Eq. (4) and equating the coefficients of like powers of $p$, we obtain the governing equation of $f_{0}(z)$ given by Eq. (6) and the governing equation of $f_{k}(z)$, as follows:

$$
\begin{aligned}
& L\left(f_{1}(z)\right)=h_{1} N_{0}\left(f_{0}(z)\right), \quad B\left(f_{1}, \frac{d f_{1}}{d z}\right)=0 \\
& L\left(f_{k}(z)-f_{k-1}(z)\right)=h_{k} N_{0}\left(f_{0}(z)\right)+\sum_{i=1}^{k-1} h_{i}\left[L\left(f_{k-i}(z)\right)+N_{k-i}\left(f_{0}(z), f_{1}(z), \ldots, f_{k-i}(z)\right)\right] \\
& B\left(f_{k}, \frac{d f_{k}}{d z}\right)=0, \quad k=2,3, \ldots
\end{aligned}
$$

where $N_{m}\left(f_{0}(z), f_{1}(z), \ldots, f_{m}(z)\right)$ is the coefficient of $p^{m}$, obtained by expanding $N\left(\phi\left(z, p, h_{i}\right)\right)$ in series with respect to the embedding parameter $p$ :

$$
N\left(\phi\left(z, p, h_{i}\right)\right)=N_{0}\left(f_{0}(z)\right)+\sum_{k \geq 1} N_{k}\left(f_{0}, f_{1}, \ldots, f_{k}\right) p^{k}, \quad i=1,2, \ldots,
$$

where $\phi\left(z, p, h_{i}\right)$ is given by Eq. (8). 
It should be emphasized that $f_{k}$ for integer $k \geq 0$ are governed by the linear Eqs. (6), (9), and (10) with the linear boundary conditions that came from origin problem, which can be solved easily.

The convergence of the series (8) depends upon the auxiliary functions $h_{1}, h_{2}, \ldots$ If it is convergent at $p=1$, one has

$$
f\left(z, h_{i}\right)=f_{0}(z)+\sum_{k \geq 1} f_{k}\left(z, h_{i}\right)
$$

Generally, the solution of Eq. (3) can be determined approximately in the form:

$$
f^{(m)}\left(z, h_{i}\right)=f_{0}(z)+\sum_{k=1}^{m} f_{k}\left(z, h_{i}\right), \quad i=1,2, \ldots, m .
$$

Substituting Eq. (13) into Eq. (3) it gives the following residual

$$
R\left(z, h_{i}\right)=L\left(f^{(m)}\left(z, h_{i}\right)+g(z)+N\left(f^{(m)}\left(z, h_{i}\right)\right)\right), \quad i=1,2, \ldots, m .
$$

If $R\left(z, h_{i}\right)=0$, then $f^{(m)}\left(z, h_{i}\right)$ happens to be the exact solution. However, such case will not occur for problems that consists of nonlinear, though we can minimize the functional

$$
J\left(C_{i j}\right)=\int_{a}^{b} R^{2}\left(z, h_{i}\right) d z, \quad i=1,2, \ldots, m ; \quad j=1,2, \ldots
$$

where $a$ and $b$ are two values, depending on the given problem. The unknown convergence-control parameters $C_{i j}, i=1,2, \ldots, m ; j=1,2, \ldots$ can be optimally identified from the conditions

$$
\frac{\partial J}{\partial C_{i j}}=0
$$

With these convergence-control parameters known, the approximate solution (of order $m$ ) (13) is well-determined. Furthermore, the convergence-control parameters $C_{i j}$ can be obtained using the methods such as Galerkin, Ritz, least square or collocation.

It is easy to observe that so-called homotopy perturbation method (HPM) is a special case of Eq. (4) when $H(p)=-p$, and on the other hand, HAM is another special case of Eq. (4) when $H(p)=-p h$ (where the parameter $h$ is chosen from so-called $h$-curves), and they can all be used to determine the parameters $C_{i j}$. While an important feature of the OHAM is that using Eq. (16), a minimization of errors is obtained.

\section{Analytical solution of Falkner-Skan equation}

The auxiliary function $H(p, z)$, can be chosen as many ways as possible. Using the conditions given in (2), we choose the initial approximation $f_{0}(z)$ as

$$
f_{0}(z)=z-\frac{1}{\beta k}+\frac{1}{\beta k} e^{-\beta k z},
$$


where $k$ is a positive convergence parameter. Then from Eqs. (2), (6) and (17), the linear operator $L$ was chosen as follows:

$$
L(f(z))=f^{\prime \prime \prime}+k f^{\prime \prime} .
$$

while, the nonlinear operator $N$ takes the form:

$$
\begin{aligned}
N(z, p)= & f_{0}^{\prime \prime \prime}+f_{0} f_{0}^{\prime \prime}+\beta\left(1-f_{0}^{\prime 2}\right)+p\left[f_{1}^{\prime \prime \prime}+f_{0} f_{1}^{\prime \prime}+f_{1} f_{0}^{\prime \prime}-2 \beta\left(f_{0}^{\prime} f_{1}^{\prime}\right)\right] \\
& +p^{2}\left[f_{2}^{\prime \prime \prime}+f_{0} f_{2}^{\prime \prime}+f_{1} f_{1}^{\prime \prime}+f_{2} f_{0}^{\prime \prime}-2 \beta\left(f_{0}^{\prime} f_{2}^{\prime}+f_{1}^{\prime 2}\right)\right]+\cdots
\end{aligned}
$$

From Eq. (19) we equate the coefficients of like powers of $p$ to obtain

$$
\begin{aligned}
& p^{0}: N_{0}\left(f_{0}\right)=f_{0}^{\prime \prime \prime}+f_{0} f_{0}^{\prime \prime}+\beta\left(1-f_{0}^{\prime 2}\right), \\
& p^{1}: N_{1}\left(f_{0}, f_{1}\right)=f_{1}^{\prime \prime \prime}+f_{0} f_{1}^{\prime \prime}+f_{1} f_{0}^{\prime \prime}-2 \beta\left(f_{0}^{\prime} f_{1}^{\prime}\right), \\
& p^{2}: N_{2}\left(f_{0}, f_{1}, f_{2}\right)=f_{2}^{\prime \prime \prime}+f_{0} f_{2}^{\prime \prime}+f_{1} f_{1}^{\prime \prime}+f_{2} f_{0}^{\prime \prime}-2 \beta\left(f_{0}^{\prime} f_{2}^{\prime}+f_{1}^{\prime 2}\right) .
\end{aligned}
$$

Now substituting Eq. (17) into Eq. (20), we obtain:

$$
N_{0}\left(f_{0}\right)=\left(k \beta z-k^{2} \beta^{2}+2 \beta-1\right) e^{-k \beta z}+(1-\beta) e^{-2 k \beta z} .
$$

Choosing $m=1$ into Eq. (7) and by applying Eq. (23), the auxiliary function $H(p, z)$ can be written in the form, $H(p, z)=p h_{1}\left(z, C_{1 i}\right)$ where $p h_{1}\left(z, C_{1 i}\right)$ is chosen in such a way that $N_{0}$ and $N_{0} h_{1}$ can be in the same form, and hence we can consider:

$$
H(p, z)=p\left(C_{11}+C_{12} e^{-k \beta z}+C_{13} e^{-2 k \beta z}\right),
$$

where $C_{11}, C_{12}, C_{13}$ and $k$ are convergence-control parameters to be determined.

Here, Eq. (9) becomes:

$$
\begin{aligned}
& f_{1}^{\prime \prime \prime}+k f_{1}^{\prime \prime}=\left(C_{11}+C_{12} e^{-k \beta z}+C_{13} e^{-2 k \beta z}\right)\left[\left(k \beta z-k^{2} \beta^{2}+2 \beta-1\right) e^{-k \beta z}+(1-\beta) e^{-2 k \beta z}\right], \\
& f_{1}(0)=f_{1}^{\prime}(0)=f_{1}^{\prime}(\infty)=0 .
\end{aligned}
$$

From now on, for simplification we will write $C_{1}, C_{2}$ and $C_{3}$ instead of $C_{11}, C_{12}$ and $C_{13}$, respectively.

Now the solution of Eq. (25) takes the form:

$$
f_{1}(z)=\frac{1}{108 k^{3}\left(k^{3}-12\right)}\left[\begin{array}{l}
-1296 k^{5} C_{1}-324 k^{5} C_{2}-144 k^{5} C_{3}+3888 k^{3} C_{1}+648 k^{3} C_{2}+240 k^{3} C_{3} \\
+\left(-6 k^{4} z C_{3}+72 k z C_{3}+6 k^{5} C_{3}-13 k^{3} C_{3}-72 k^{2} C_{3}+156 C_{3}\right) e^{-3 k z}
\end{array}\right]
$$


The first-order approximate solution (13) is

$$
f^{(1)}(z)=f_{0}(z)+f_{1}(z)
$$

Substituting Eqs. (17) and (26) into Eq. (27) we obtain:

$$
\begin{aligned}
f^{(1)}(z)= & \frac{1}{108 k^{3}\left(k^{3}-12\right)}\left[\begin{array}{l}
-1296 k^{5} C_{1}-324 k^{5} C_{2}-144 k^{5} C_{3}+3888 k^{3} C_{1}+648 k^{3} C_{2}+240 k^{3} C_{3} \\
+\left(-6 k^{4} z C_{3}+72 k z C_{3}+6 k^{5} C_{3}-13 k^{3} C_{3}-72 k^{2} C_{3}+156 C_{3}\right) e^{-3 k z}
\end{array}\right] \\
& +z-\frac{1}{\beta k}+\frac{1}{\beta k} e^{-\beta k z}
\end{aligned}
$$

The residual (14) becomes in this case:

$$
R\left(z, C_{1}, C_{2}, C_{3}, k\right)=f^{(1)^{\prime \prime \prime}}(z)+f^{(1)}(z) f^{(1)^{\prime \prime}}(z)+\beta\left(1-f^{(1)^{\prime 2}}(z)\right) .
$$

From Eq. (16), this becomes $\frac{\partial J}{\partial C_{i}}=\frac{\partial J}{\partial k}=0, \quad i=1,2,3$ we obtain:

$$
\begin{aligned}
& C_{1}=0.00186322166, \quad C_{2}=0.4026020376, \quad C_{3}=-0.02385423487, \\
& \quad k=1.231562651244 .
\end{aligned}
$$

Sequel to the presentations made in Figs. 1 and 2, where the results for this study appears perfectly matched that of Bararnia et al. (2012) from which the increasing order of Padé approximation on the velocity profile shows a significant effect, as depicted in Fig. 1. While Fig. 2, has indicated the good agreements between the present studies with numerical solution.

\section{Conclusions}

In this study, a new analytical technique is proposed to get a solution of Falkner-Skan equation. The method gives a desired analytical solution of the 2D laminar, incompressible viscous fluid flow over a semi-infinite wedge. The validation of this study with numerical and Bararnia et al. (2012), as shown in Figs. 1 and 2, show the excellence and

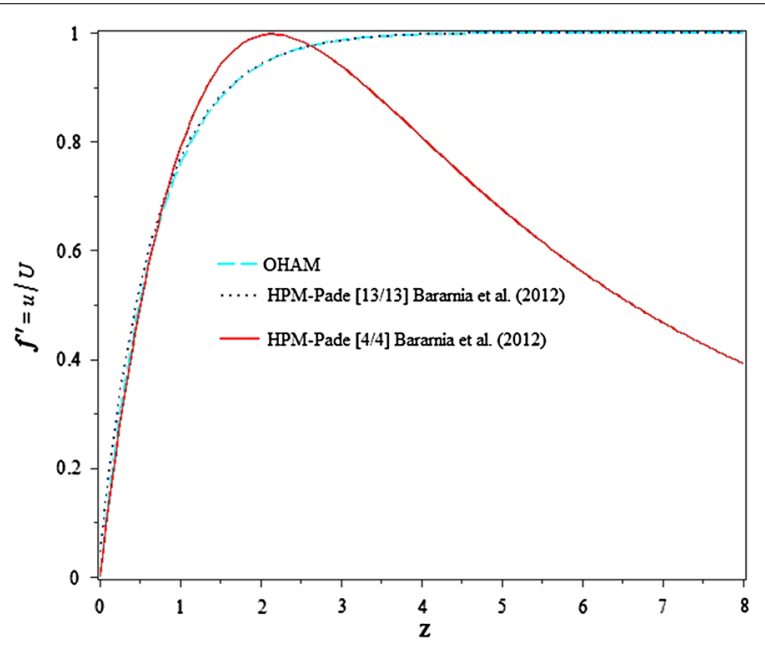

Fig. 1 Comparison of OHAM solution with HPM-Padé solution and the effect of increasing the order of its approximation, on the velocity profile for $\beta=1$. 


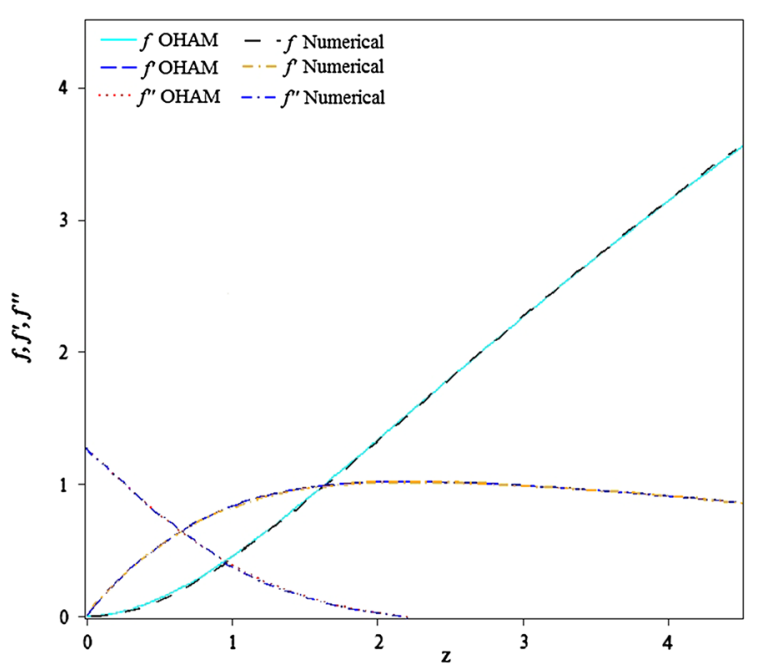

Fig. 2 Comparison of OHAM with numerical solutions for $\beta=1$.

capability of OHAM towards handling both linear and nonlinear problems. However, an excellent agreement between those methods has achieved, because the values we obtained for $f^{\prime \prime}(0)=1.23140046$ at $\beta=1$, agreed with $f^{\prime \prime}(0)=1.23150$ (Bararnia et al. 2012) and $f^{\prime \prime}(0)=1.2325$ (Rajagopal et al. 1983) all at $\beta=1$. Despite the fact that only first-order approximation been generated.

Authors' contributions

All authors have contributed to the development of this study equally. All authors read and approved the final manuscript.

\section{Author details}

Centre for Research in Computational Mathematics Faculty of Science, Technology and Human Development, Universiti Tun Hussein Onn Malaysia, 86400 Batu Pahat, Johor, Malaysia. ${ }^{2}$ Department of Mathematics, School of Science and Technology, Federal Polytechnic Bauchi, P.M.B. 0231, Off Dass Road, Bauchi, Nigeria.

\section{Acknowledgements}

The author would like to acknowledge the financial aid received from Universiti Tun Hussein Onn Malaysia the Grant GIPS/2014/UTHM/U191 and U136.

\section{Competing interests}

The authors declare that they have no competing interests.

Received: 15 December 2015 Accepted: 12 April 2016

Published online: 26 April 2016

\section{References}

Abbasbandy S, Hayat T (2009) Solution of the MHD Falkner-Skan flow by homotopy analysis method. Commun Nonlinear Sci Numer Simul 14:3591-3598

Abdulhameed M, Saleh H, Hashim I, Roslan R (2015) Radiation effects on two-dimensional MHD Falkner-Skan wedge flow. Appl Mech Mater 773-774:368-372

Alizadeh E, Farhadi M, Sedighi K, Ebrahimi-Kebria HR, Ghafourian A (2009) Solution of the Falkner-Skan equation for wedge by Adomian Decomposition Method. Commun Nonlinear Sci Numer Simul 14:724-733

Asaithambi NS (1997) A numerical method for the solution of the Falkner-Skan equation. Appl Math Comput 81:259-264 Bararnia H, Ghasemi E, Soleimani S, Ghotbi AR, Ganji DD (2012) Solution of the Falkner-Skan wedge flow by HPM-Padé method. Adv Eng Softw 43:44-52

Falkner VM, Skan SW (1931) Some approximate solutions of the boundary layer equations. Philos Mag 12(80):865-896

Fang T, Yao S, Zhang J, Zhong Y, Tao H (2012) Momentum and heat transfer of the Falkner-Skan flow with algebraic decay: an analytical solution. Commun Nonlinear Sci Numer Simul 17:2476-2488

Hartree DH (1937) On an equation occurring in Falkner and Skan's approximate treatment of the equations of the boundary layer. Proc Camb Philos Soc 33(Part II):223-239 
Hayat T, Hussain M, Nadeem S, Mesloudn S (2011) Falkner-Skan wedge flow of a power-law fluid with mixed convection and porous medium. Comput Fluids 49:22-28

Herisanu N, Marinca V, Madescu Gh (2015) An analytical approach to non-linear dynamical model of a permanent magnet synchronous generator. Wind Energy 18:1657-1670

Hsiao K-L (2011) MHD mixed convection for viscoelastic fluid past a porous wedge. Int J Non Linear Mech 46:1-8

Ishak A, Nazar R, Pop I (2009) MHD boundary-layer flow of a micropolar fluid past a wedge with constant wall heat flux. Commun Nonlinear Sci Numer Simul 14:109-118

Kuo B-K (2005) Heat transfer analysis for the Falkner-Skan wedge flow by the differential transformation method. Int J Heat Mass Transf 48:5036-5046

Marinca V, Herisanu N (2008) Application of optimal homotopy asymptotic method for solving nonlinear equations arising in heat transfer. Int Commun Heat Mass Transfer 35:710-715

Marinca V, Herisanu N (2014) The optimal homotopy asymptotic method for solving Blasius equation. Appl Math Comput 231:134-139

Nayfeh AH, Mook DT (1979) Nonlinear oscillations. Willey, New York

Pantokratoras A (2006) The Falkner-Skan flow with constant wall temperature and variable viscosity. Int J Thermal Sci 45:378-389

Parand K, Rezaei AR, Ghaderi SM (2011) An approximate solution of the MHD Falkner-Skan flow by Hermite functions pseudospectral method. Commun Nonlinear Sci Numer Simul 16:274-283

Rajagopal KR, Gupta AS, Na TY (1983) A note on the Falkner-Skan flows of a non-Newtonian fluid. Int J Non Linear Mech 18:313-320

Rosales-Vera M, Valencia A (2010) Solutions of Falkner-Skan equation with heat transfer by Fourier series. Int Commun Heat Mass Transf 37:761-765

Yacob NA, Ishak A, Pop I (2011) Falkner-Skan problem for a static or moving wedge in nanofluids. Int J Thermal Sci 50:133-139

Yao B (2009) Series solution of the temperature distribution in the Falkner-Skan wedge flow by the homotopy analysis method. Eur J Mech B Fluids 28:689-693

Yao B, Chen J (2009) Series solution to the Falkner-Skan equation with stretching boundary. Appl Math Comput 208:156-164

Zhu S, Wu Q, Cheng X (2009) Numerical solution of the Falkner-Skan equation based on quasilinearization. Appl Math Comput 215:2472-2485

\section{Submit your manuscript to a SpringerOpen ${ }^{\circ}$ journal and benefit from:}

- Convenient online submission

\section{- Rigorous peer review}

- Immediate publication on acceptance

- Open access: articles freely available online

- High visibility within the field

- Retaining the copyright to your article

Submit your next manuscript at $\boldsymbol{\nabla}$ springeropen.com 